\title{
Spontaneous Rupture of a Right Atrial Angiosarcoma and Cardiac Tamponade
}

\author{
Ricardo Barros Corso, Nadja Kraychete, Sidnei Nardeli, Rilson Moitinho, Cristiano Ourives, \\ Rosenbert Mamedio da Silva, Ricardo Eloy Pereira
}

Salvador, BA - Brazil

\begin{abstract}
Primary cardiac angiosarcoma is a rare disease of difficult diagnosis and poor prognosis frequently associated with recurring hemopericardium. We report the case of a 30-year-old female with a right atrial angiosarcoma and spontaneous rupture to the pericardial cavity, who was diagnosed during an emergency exploratory thoracotomy, whose indication was cardiac tamponade. This is the 8 th case reported in the literature. Clinical findings are discussed and a literature review is provided.
\end{abstract}

Angiosarcoma is a tumor of mesenchymal origin and accounts for approximately $25 \%$ of the malignant tumors of the heart. It preferably appears in the right atrium between the 3 rd and 5th decades of life. It is characterized by early local and systemic dissemination, which restricts the indication for surgical resection to a small number of patients. The use of adjuvant chemotherapy and radiation therapy is controversial, due to the poor patient prognosis because of the disease, mean life expectancy being just 6 months ${ }^{1}$.

Spontaneous rupture of an angiosarcoma is extremely rare, and only 7 other cases have been reported in the literature. We report a case with this severe complication, preceded by repeated pericardial effusion and no preoperative suspicion of tumor. The clinical evolution is described and a literature review provided ${ }^{2,3}$.

\section{Case Report}

The patient was a 30-year-old black female reporting deterioration of her general condition, weight loss, and daily recurring fever for 9 months. On admission to our service, she also reported having dyspnea in the 4 preceding months.

The clinical and laboratory investigation showed ane-

Serviço de Cirurgia Cardíaca Cardiocirúrgica, Serviço de Cardiologia Clínica Cardiointensiva, Hospital Santa Izabel da Santa Casa de Misericórdia

Mailing address: Ricardo Barros Corso - Rua Altino Serberto de Barros, 345/1302 Cep 41810-570 - Salvador, BA, Brazil - E-mail: ricardocorso@ig.com.br. English version by Stela Maris C. e Gandour mia, enlargement of the cardiac area on simple chest radiography, and voluminous pericardial effusion on transthoracic echocardiography. The other examinations were within normal parameters.

The patient underwent pericardiocentesis, and 2000 $\mathrm{mL}$ of a frankly hemorrhagic fluid were withdrawn. During the same admission, open pericardial drainage was required due to occurrence of new effusion with hemodynamic repercussions. Laboratory and cytological analysis of the pericardial fluid was inconclusive. The patient was discharged from the hospital for follow-up and ambulatory investigation with improvement in her clinical condition, but with no diagnostic confirmation of the cause of the disease.

The patient was readmitted 4 months later with significant worsening of her general condition and continuation of her previous complaints, among which, dyspnea and isolated episodes of hemoptysis predominated. On physical examination, the patient was extremely pale, with signs of decompensated right heart failure and multiple rales in both lungs. A slight systolic murmur in the mitral area and a precordial thrill could be heard.

The laboratory tests showed hypochromic microcytic anemia, anisocytosis, polychromatophilia, presence of schizocytes and target cells, severe reduction in the number of platelets, slight leukocytosis with no shift to the left, hypoalbuminemia, hypoprothrombinemia, and generalized coagulation disorder. The electrocardiogram showed sinus tachycardia and right ventricular overload. The chest radiography showed multiple nodular images in both lungs and bilateral pleural effusion. The transthoracic echocardiogram revealed moderate septate pericardial effusion with no other intracardiac findings.

Cardiopulmonary deterioration occurred 2 days after admission, and the patient was referred to the ICU. A new transthoracic echocardiography revealed an increase in the effusion volume and signs of cardiac tamponade. The patient was referred for open pericardial drainage to be performed with her under general anesthesia. The patient experienced hemodynamic instability from the time of the induction of 
anesthesia, and a voluminous hemorrhage was observed after a pericardial opening through a subxiphoid incision, which was followed by hypovolemic shock. Immediate median sternotomy was performed with extension of the first incision followed by longitudinal pericardiotomy. A rupture in the right atrial free wall measuring $3 \times 3 \mathrm{~cm}$ was identified, close to the junction of the atrium and the superior vena cava in an area of necrotic tumoral tissue extending throughout most of the atrium. Multiple pericardial implants with the same appearance were identified. The rupture was sutured with temporary clamping of the venae cava, and massive volemic replacement and cardiopulmonary resuscitation were provided. After transitory recovery of the vital signs, the patient experienced refractory shock and died (fig. 1).

Intracardiac inspection revealed extensive tumoral involvement in most of the right atrium with the same wall thickness. The interatrial septum, valves, and other cardiac chambers had no abnormalities.

The pleural cavities were opened for pulmonary biopsy, and multiple subpleural hemorrhagic implants were identified in both lungs, in addition to bilateral voluminous hemorrhagic pleural effusion.

The histopathologic study revealed disseminated angiosarcoma involving the heart, pericardium, and lungs (fig. 2).

\section{Discussion}

Primary heart tumors are extremely rare, with an incidence of $0.0017 \%$ in autopsy studies reported by the American Medical Association. Cardiac metastases are 20 to 40 times more frequent than are primary heart tumors. Only $25 \%$ of the cardiac tumors are malignant, sarcomas being the most common. Angiosarcomas account for 25-30\% of those tumors. Of the 24 cases reported by Donsbeck et al ${ }^{4}$ with immunohistochemical confirmation, 9 were undifferentiated sarcomas, 6 were angiosarcomas, 6 were leiomyosarcomas, and 3 had other diagnoses.

Angiosarcomas have already been called hemangio-

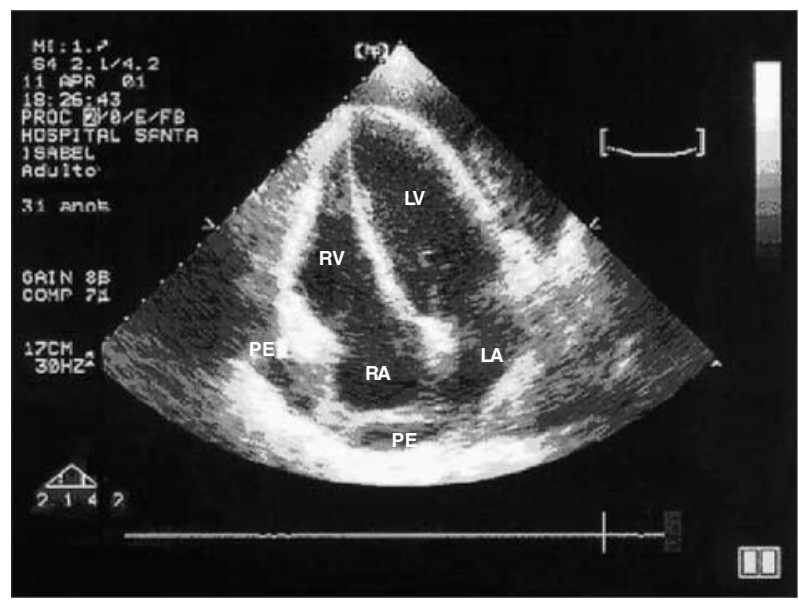

Fig. 1 - Transthoracic echocardiographic image of a voluminous pericardial effusion with septations and signs of cardiac tamponade. RV-right ventricle; LV- left ventricle; LA- left atrium; RA- right atrium; PE- pericardial effusion.

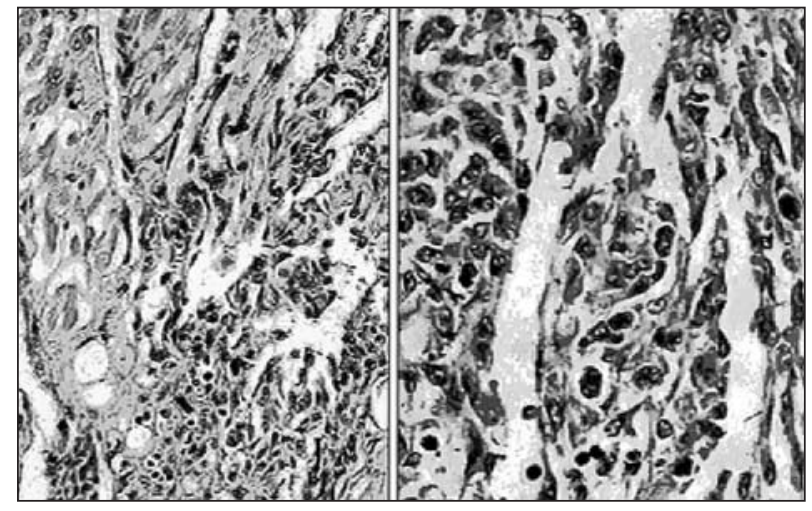

Fig. 2 - Histological sections of a myocardial fragment stained with hematoxylin and eosin. Note the fusiform cells with atypical vesicular nuclei delimiting slit vascular structures characteristic of angiosarcoma.

sarcoma, hemangioendothelioma, malignant hemangioendothelioma, angioendothelial sarcoma, hemangioendothelial sarcoma, malignant hemangioma, and hemangioendothelial blastoma.

An angiosarcoma of the heart is considered primary when no history or concomitant evidence is present of tumors in the soft tissue, bone, or subcutaneous tissue. In a case series of 366 cases, $3 \%$ of the tumors originated from the heart or great vessels, or both. Angiosarcoma of the heart involves almost exclusively the right atrium, but has already been reported in other cardiac chambers ${ }^{5,6}$.

The symptoms resulting from primary tumors of the heart are usually late and more related to their location than to their histological type, which makes the early diagnosis difficult, impairing the efficacy of treatment. Myocardial infiltration by the tumor triggers arrhythmias and, more rarely, conduction disorders. Impairment of myocardial contractility may simulate restrictive or dilated hypertrophic cardiomyopathy. Pulmonary arterial hypertension secondary to repetitive tumoral embolization and compression of the right ventricular outflow tract may trigger right heart failure.

Angiosarcomas of the heart grow rapidly, usually within the myocardial wall, which makes their diagnosis through different imaging methods difficult. They are characterized by friability and a tendency towards bleeding. They are often associated with pericardial effusion and cardiac tamponade. Myocardial rupture due to tumoral infiltration and necrosis of the wall may occur, multiseptate hemopericardium being the most frequent echocardiographic finding in the few cases reported ${ }^{2,6}$.

The symptoms resulting from mechanical cardiac impairment are usually preceded by malaise, fever, weight loss, fatigue, and anemia that occur weeks or months before symptom onset. Dyspnea, productive cough, and hemoptysis suggest pulmonary metastases. The most frequent sites of metastases are the pericardium, lungs, mediastinal lymph nodes, and vertebrae, and metastases are usually present in 66 to $89 \%$ of the patients at the time of diagnosis ${ }^{7,8}$.

The diagnosis of angiosarcoma is usually difficult and late, despite the different methods used. Many cases have been confirmed only on thoracotomy for the treatment 
of repetitive pericardial effusion or on autopsy. Echocardiography is the most frequently used method, the transesophageal technique being the preferred one. The presence of cavitary-pericardial fistula in cases of cardiac rupture has already been identified by that method ${ }^{2}$.

Patients with the tumoral form may have their diagnosis suggested by computed tomography or nuclear magnetic resonance. No method of myocardial biopsy is advisable, due to the characteristic friability of angiosarcoma and its predisposition to hemorrhaging ${ }^{6}$.

The treatment of angiosarcoma is controversial due to the poor prognosis in most patients. Surgical resection is indicated when no evidence of metastases exists and when myocardial resection is reparative 9 .

Chemotherapy and radiation therapy may be indicated as adjuvant or preferential therapies, but their use is usually limited due to the poor physical condition of the patient. Survival ranges from 6 to 9 months regardless of the treatment chosen. Nakamichi et al ${ }^{10}$ reported the case of a patient diagnosed with cardiac angiosarcoma at the age of 8 years, who was treated with an intensive and multidisciplinary approach for 2 years and has an excellent quality of life 53 months after the diagnosis. Cardiac transplantation has already been performed in cases with an earlier and more favorable diagnosis, but the use of immunosuppressant drugs is a limiting factor, because it predisposes one to tumor recurrence and metastases. Cardiomyoplasty is a surgical alternative when partial ventricular resection is required.

Primary cardiac angiosarcoma should be considered in the differential diagnosis of recurrent hemopericardium, and septation of the effusion, as in our case, suggests rupture of the free wall.

\section{References}

1. Frota FJD, Luchese FA, Leães P, Valente LA, Vieira MS, Blacher C. Angiossarcoma cardíaco primário: um dilema terapêutico. Arq Bras Cardiol 2002;78:586-8.

2. Ohri SK, Nihoyannopoulos P, Taylor KM, Keogh BE. Angiosarcoma of the heart causing cardiac rupture: a cause of hemopericardium. Ann Thorac Surg 1993;55:525-8

3. Mukohara N, Tobe S, Azami T. Angiosarcoma causing cardiac rupture. Jpn J Thorac Cardiovasc Surg 2001;49:516-8.

4. Donsbeck AV, Ranchere D, Coindre JM, Le Gall F, Cordier JF, Loire R. Primary cardiac sarcomas: an immunohistochemical and grading study with long-term follow-up of 24 cases. Histopathology 1999;34:295-304.

5. Herrmann MA, Shakermen RA, Edwards WD, ShubC, SchaffHV. Primary cardiac angiosarcoma: a clinicopathologic study of six cases. J Thorac Cardiovasc Surg 1992;103:655-65.
6. Oshima K, Ohtaki A, Motoi K, et al. Primary cardiac angiosarcoma associated with cardiac tamponade. Jpn Circ J 1999;63:822-4

7. Biniwale RM, Pathare HP, Aggrawal N, Tendolkar AG, Deshpande J, Sivaraman A. Cardiac sarcomas: is tumor debulking justifiable therapy? Asian Cardiovasc Thorac Ann 1999;7:52-5.

8. Shapiro S, Scott J, Kaufman K. Metastatic cardiac angiosarcoma of the cervical spine: case report. Spine 1999;24:1156-9

9. Mcfadden PM, Ochsner JL. Atrial replacement and tricuspid valve reconstrution after angiosarcoma resection. Ann Thorac Surg 1997;64:1164-6.

10. Nakamichi T, Fukuda T, Suzuki T, Kaneko T, Morikawa Y. Primary cardiac angiosarcoma: 53 months' survival after multidisciplinary therapy. Ann Thorac Surg 1997;63:1160-1. 